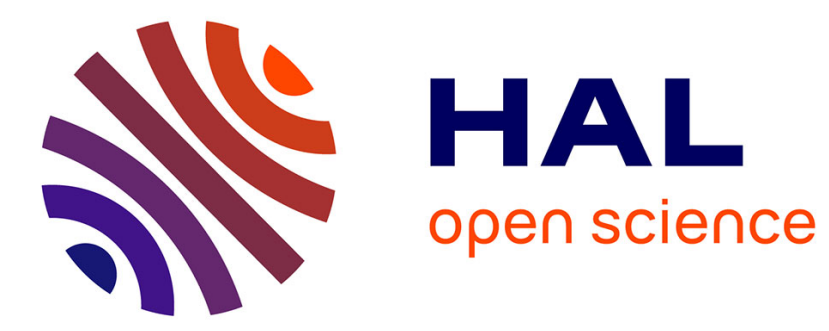

\title{
Techniques de hautes pressions à très basse température
}

\author{
G. Malfait, D. Jerome
}

\section{To cite this version:}

G. Malfait, D. Jerome. Techniques de hautes pressions à très basse température. Revue de Physique Appliquée, 1969, 4 (4), pp.467-470. 10.1051/rphysap:0196900404046700 . jpa-00243312

\section{HAL Id: jpa-00243312 https://hal.science/jpa-00243312}

Submitted on 1 Jan 1969

HAL is a multi-disciplinary open access archive for the deposit and dissemination of scientific research documents, whether they are published or not. The documents may come from teaching and research institutions in France or abroad, or from public or private research centers.
L'archive ouverte pluridisciplinaire $\mathbf{H A L}$, est destinée au dépôt et à la diffusion de documents scientifiques de niveau recherche, publiés ou non, émanant des établissements d'enseignement et de recherche français ou étrangers, des laboratoires publics ou privés. 


\title{
TEGHNIQUES DE HAUTES PRESSIONS A TRÈS BASSE TEMPÉRATURE ( $\left.{ }^{1}\right)$
}

\author{
Par G. MALFAIT et D. JEROME, \\ Laboratoire de Physique du Solide $\left({ }^{2}\right)$, Faculté des Sciences, 9 I-Orsay.
}

(Reçu le 2y mai 1c69.)

\begin{abstract}
Résumé. - Nous avons mis au point un appareil de pression hydrostatique pour travailler à la température de $4,2 \circ \mathrm{K}$ jusqu'à $17 \mathrm{kbars}$. Nous donnons les descriptions de la bombe de pression, des systèmes de fermeture et des passages des fils électriques à travers les obturateurs.

Les montages que nous proposons sont d'un usinage aisé et ont prouvé leur efficacité sur un grand nombre d'expériences sous pression à basse température.
\end{abstract}

Abstract. - We shall give in this paper a description of a high hydrostatic pressure and low temperature apparatus for studies to $17 \mathrm{kbars}$ and $4.2 \mathrm{~K}$.

Details about pressure vessel, low temperature seals and electrical leads are given. Some techniques facilitating machining of the equipment are given. This equipment has proved to be effective in a number of pressure experiments.

I. Introduction. - Nous allons présenter dans cet article quelques développements techniques que nous avons été amenés à faire dans le but de réaliser des mesures sous haute pression hydrostatique à la température de l'hélium liquide.

Cet article n'est nullement une revue de tous les dispositifs expérimentaux imaginés jusqu'à ce jour. Nous désirons seulement donner la description de montages mis au point au laboratoire et qui ont déjà fait leurs preuves sur un nombre important d'expériences. Nous donnerons aussi des méthodes permettant la réalisation simple et rapide de parties de l'appareil particulièrement délicates. On peut dire que la plupart des dispositifs expérimentaux de physique sous pression avaient été inventés par P. W. Bridgman [1]. Ge que nous avons fait a été de perfectionner ces dispositifs, de les adapter au travail à très basse température et surtout de mettre au point un équipement d'utilisation sûre et reproductible.

Beaucoup d'expérimentateurs de physique sous pression savent combien les expériences de pression hydrostatique au-dessus de $15 \mathrm{kbars}$ sont encore aléatoires.

Avec l'appareil de pression que nous utilisons, le fluide transmetteur de pression est soit de l'hélium, soit un mélange $1 / 2$ alcool isoamylique- $1 / 2$ pentane. Le générateur de pression est identique à celui décrit par Langer et Warschauer [2]. L'hélium gazeux d'un cylindre à 180 bars est amené à 2 kbars dans un multiplicateur de rapport 1; ensuite un multiplicateur de rapport 76 comprime le gaz jusqu'à la pres-

(1) Travail effectué avec l'aide du C.N.R.S. et de la D.R.M.E., contrat no 403-67.

${ }^{(2)}$ Laboratoire associé au C.N.R.S. sion nécessaire à la mesure. Cette partie de l'appareil a été construite par la société Basset, 92-Sèvres. Un tube capillaire souple établit la liaison entre le multiplicateur haute pression et la bombe de mesure. Pour les expériences en liquide, seul le multiplicateur haute pression est utilisé.

La bombe de mesure est située à l'intérieur d'un cryostat, dont la température est réglable de $300^{\circ} \mathrm{K}$ jusqu'à 4,2 oK. Les parties de l'appareil que nous allons décrire sont :

1) La bombe de mesure haute pression à basse température;

2) Le système de joints permettant l'étanchéité des obturateurs de fermeture de la bombe;

3) Les passages étanches et isolés électriquement des fils à travers l'obturateur.

II. Bombe haute pression. - Comme l'indique la figure 1, la bombe est à deux enveloppes, d'une part pour augmenter le domaine des pressions de travail, et d'autre part pour des raisons de sécurité. L'âme est en acier Vasco-Max 350 traité à 40-44 Rockwell C.

Avant emmanchage, le diamètre extérieur de l'âme était supérieur de 25/100 de $\mathrm{mm}$ au diamètre intérieur de la frette. La cavité de mesure à l'intérieur de la bombe est un cylindre de $\varnothing 13,5 \times 35 \mathrm{~mm}$. Comme il est visible sur la figure 1, la bombe est fermée par deux obturateurs, l'un contient le capillaire haute pression et l'autre les passages électriques.

Cette bombe de pression est montée environ 50 fois en pression, dont 4 fois à la pression de 17,5 kbars.

Habituellement, le refroidissement de la bombe a lieu après la mise sous pression. Toutefois, en gaz hélium, nous avons pu changer la pression dans de 

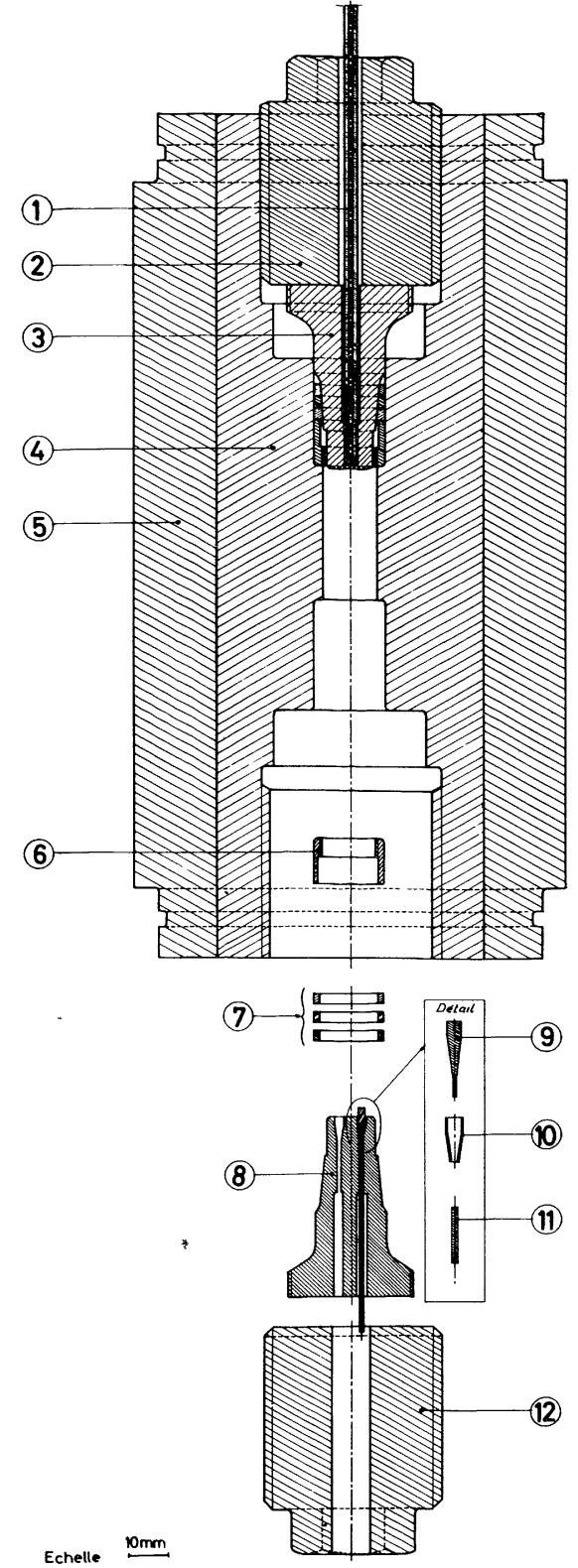

FIG. 1. - Vue de la bombe de pression et de ses obturateurs : (1), Capillaire haute pression; (2), Vis de l'obturateur du capillaire; (3) Obturateur du capillaire; (4), Ame en Vasco-Max 350; (5), Frette en Vasco-Jet $1000 ;(6)$, Bague d'extraction des joints; (7), Joints ; (8), Obturateur avec passages électriques ; (9), Détails d'un passage électrique conique avec (10) Isolement d'araldite et Fil (11) ; (12) Vis de l'obturateur des passages électriques.

très larges limites à $77 \mathrm{oK}$. Cela semble indiquer que les aciers Maraging ne sont pas trop fragiles à basse température.

Jusqu'à la pression de 17,5 kbars, nous n'avons jamais observé de déformations permanentes sur les diamètres internes de la bombe. Nous pensons donc que la bombe de la figure 1 devrait pouvoir contenir des pressions supérieures à 20 kbars.

En conclusion, les aciers Maraging dont les propriétés à $4,2{ }^{\circ} \mathrm{K}$ sont encore peu connues semblent bien adaptés au problème des bombes de pression à basse température [3].

III. Obturateurs et joints. - La fermeture d'une bombe de pression est un problème délicat surtout en gaz à une pression supérieure à 10 kbars.

Le principe le plus connu est celui de l'obturateur de type " champignon » à aire non supportée que P. W. Bridgman a adapté aux hautes pressions [4]. Sans revenir sur la description de la fermeture décrite dans la précédente référence, on peut seulement signa-

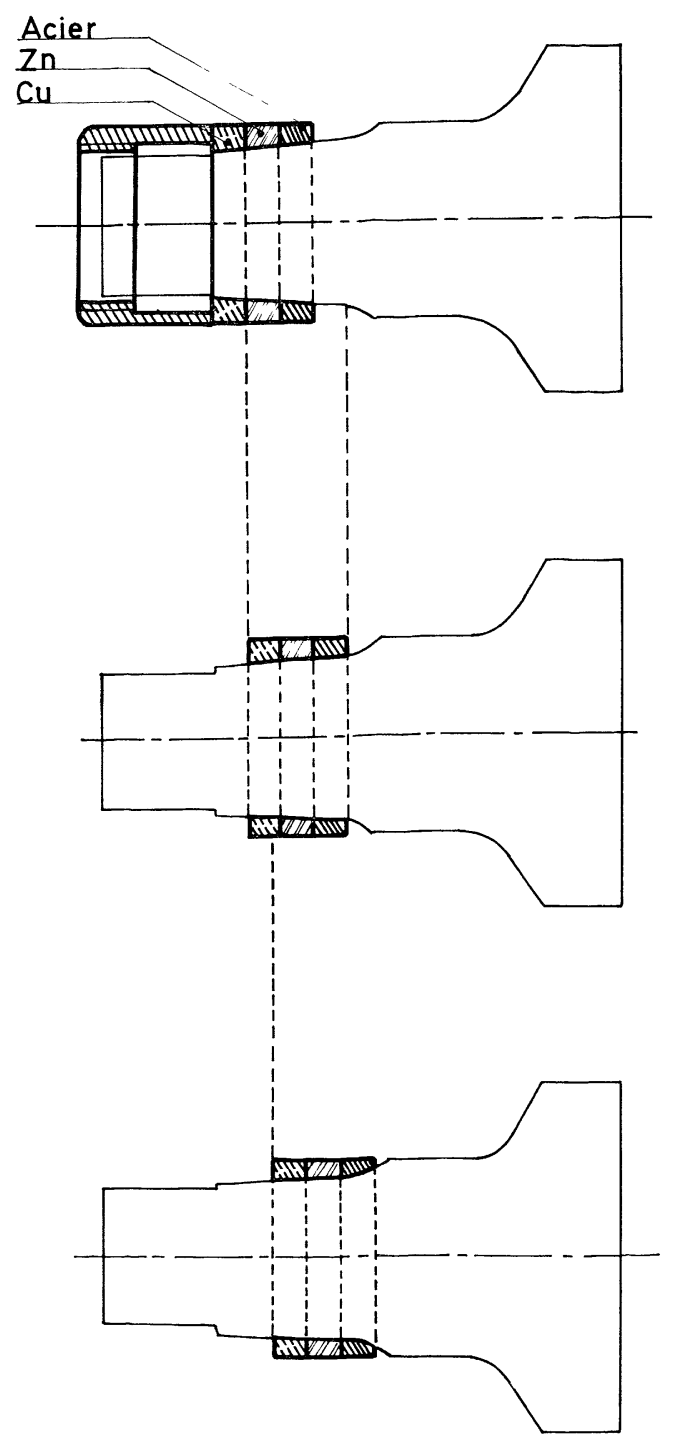

(a)
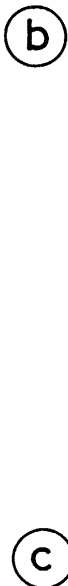

FIG. 2. - Montage d'un obturateur et des joints d'étanchéité. 


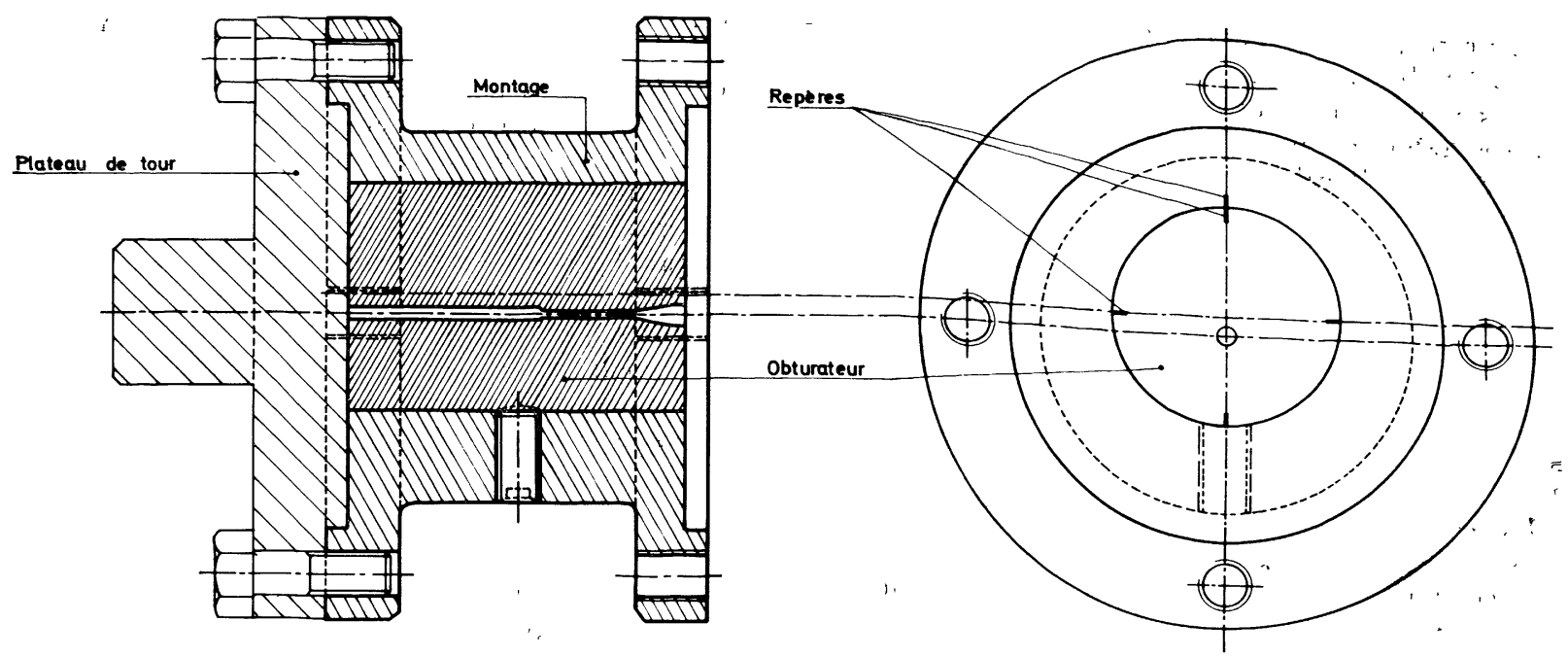

Fig. 3. - Montage excentré utilisé pour l'usinage des 4 passages électriques.

ler qu'elle présente un diamètre utilisable faible pour le passage de tubes capillaires ou de fils électriques. En outre, le phénomène de striction du « champignon » peut apparaître sous pression.

Un second principe aussi fort employé est celui de l'obturateur cylindrique à joints encastrés [5].

C'est l'obturateur à joints encastrés que nous avons modifié et perfectionné afin de le rendre d'un emploi sûr et reproductible en gaz sous très haute pression. Le plan que nous avons employé est présenté sur les figures 1 et 2. La différence avec les obturateurs à joints encastrés des références [5] est que nous avons utilisé une portée de joints coniques, afin de conserver une aire non supportée, importante même sous haute pression, lorsque les joints ont flué. L'angle du cône est de $6^{\circ}$. Le cône se raccorde à la partie cylindrique de l'obturateur par un rayon.

Les obturateurs sont en acier Maraging, VascoMax 350 exactement identique à la bombe. La partie inférieure représente un obturateur avec les passages électriques, les joints et la bague d'extraction filetée, qui permet de retirer les joints usagés. La portée des joints sur la bombe demande une finition correcte, par exemple une rectification.

Les joints utilisés sont au nombre de trois : acier Maraging à l'état adouci ou acier inoxydable, zinc et cuivre. L'intérieur est conique, l'extérieur est cylindrique. Sur la figure $2 \mathrm{a}$, avant serrage, les joints sont parfaitement ajustés à l'obturateur conique. Le jeu du diamètre extérieur par rapport à l'alésage de la portée sur la bombe est $\simeq 3 / 100 \mathrm{de} \mathrm{mm}$. L'épaisseur de chaque joint est $3 \mathrm{~mm}$. Avant serrage sur la figure $2 \mathrm{a}$, l'espace libre entre le joint d'acier et le début du rayon est $\simeq 3 \mathrm{~mm}$.

Le serrage s'effectue au moyen de la vis (12) (fig. 1 et $2 \mathrm{~b}$ ).

L'étanchéité est alors réalisée par les joints en cuivre REVUE DE PHYSIQUe APPLIQUÉE. - T. 4. No 4. DÉCEMBRE 1969. et zinc. Pendant la montée en pression, les joints fluent progressivement vers la base de l'obturateur (fig. $2 \mathrm{c}$ ). Nous avons constaté que, pour 17,5 kbars, le joint en acier ne remplit pas encore le rayon. Il y a une aire non supportée importante qui assure l'étanchéité.

Nous avons pu effectuer plusieurs montées en pression successives, en ouvrant la bombe et en laissant les joints en place entre chaque expérience. Il n'est pas nécessaire d'effectuer un violent préserrage, puisque ces joints sont « automatiques » et que le serrage est obtenu par la pression elle-même. L'extraction de l'obturateur conique s'effectue sans difficultés de même que celle des trois joints.

Nous n'avons pas rencontré de problèmes pour travailler à $4,2 \mathrm{oK}$.

IV. Les passages électriques. - Plusieurs montages ont été utilisés pour le passage des fils électriques à travers l'obturateur [2], [5], [6] et [7]. Le montage que nous allons décrire est basé sur le principe des passages coniques employés par P. W. Bridgman.

Nous allons tout d'abord exposer un procédé très simple d'exécution qui n'utilise qu'un tour à l'exclusion de toute fraiseuse.

Avant d'exécuter les travaux de tour sur l'obturateur décrit au paragraphe III, il est simple de commencer par les passages électriques. Un cylindre en acier Maraging à l'état adouci est placé dans un montage fixé sur le plateau d'un tour ( fig. 3). Le trou cylindrique du précédent montage est excentré afin de pouvoir exécuter successivement les 4 passages coniques de l'obturateur. Les cônes percés dans l'obturateur ont un angle de $16^{\circ}$ ( $f i g$. 1). Ils sont suivis d'un trou de $1 \mathrm{~mm}$ de diamètre sur $12,5 \mathrm{~mm}$ de long. L'électrode conique (9) est en acier Maraging. La gaine (10), qui assure à la fois l'étanchéité et l'isole- 
ment électrique, est en araldite chargé au talc d'épaisseur 2/10 de mm [8].

Nous avons utilisé l'araldite de préférence à la pyrophyllite habituellement utilisée par les expérimentateurs. En effet, l'araldite chargé s'usine très facilement, est étanche à l'hélium gazeux et possède une excellente résistance à la pression. On enregistre cependant un léger fluage de l'araldite sous 17,5 kbars. Il est nécessaire pour cette raison de laisser une queue d'environ $4 \mathrm{~mm}$ au bout du cône afin que l'araldite ne vienne pas sectionner le fil (11) lors du fluage.

L'isolement électrique est supérieur à $1 \mathrm{Meg}$. Un léger coup de marteau sur la base de l'électrode conique assure l'étanchéité à basse pression.

Ces passages sont insensibles à un cyclage en pression et température et sont particulièrement robustes.

En conclusion, il nous a semblé intéressant de présenter l'ensemble de la bombe et de ses obturateurs qui a déjà fait ses preuves sur environ 50 expériences sous pression à la température de l'hélium liquide. L'emploi des aciers Maraging pour l'appareillage basse température est à conseiller en raison de leur haute résistance mécanique et de leur faible fragilité à basse température.

Ce qui limite actuellement la pression dans l'appareil que nous avons décrit et la résistance du tube capillaire reliant la bombe au multiplicateur.

Étant donné que ni la bombe, ni les obturateurs ne donnent de signes de faiblesse vers 17,5 kbars, nous pensons pouvoir faire travailler ce même équipement jusque vers 20 kbars avec de meilleurs capillaires dans le futur.

Les auteurs tiennent à remercier MM. E. Fabre et $\mathrm{D}$. Le Fur pour leur participation à l'élaboration de l'appareillage de haute pression.

\section{BIBLIOGRAPHIE}

[1] Bridgman (P. W.), The Physics of High Pressure, G. Bell and Sons, London, 1958.

[2] LANGer (D.) et Warschauer (D. M.), Rev. Sci. Instrum., 1961, 32, 32.

[3] Acheté à la Société Commentryenne des Aciers Fins, Vanadium Alloys, Paris.

[4] BRIDGMaN (P. W.), loc. cit., p. 34.

WARSChaUeR (D. M.) et PAUL (W.), Rev. Sci. Instrum., 1957, 28, 62; Rev. Sci. Instrum., 1958, 29, 675.

[5] BRIDGMAN (P. W.), loc. cit., p. 37.

PaUl (W.), Benedek (G. B.) et Warschauer (D. M.), Rev. Sci. Instrum., 1959, 30, 874.
Gorce (W. S.), MCDOWELI (B.) et ScoTT (T. A.), Rev. Sci. Instrum., 1965, 36, 99.

[6] Bridgman (P. W.), loc. cit., p. 52.

[7] CORnish (R. H.) et RUOFF (A. E.), Rev. Sci. Instrum., 1961, 32, 639.

Schirber (J. E.) et ShanfeidT (D. W.), ibid., 1968, 39, 270.

MARTINSON (R. H.) et TERRY (R. E.), ibid., 1967, 38, 1330.

[8] Silitro, Paris. 